\title{
¿MÚSICA O MAGIA? LA PRESENTACIÓN DE LAS ONDAS MUSICALES DE MAURICE MARTENOT EN ESPAÑA
}

\author{
MUSIC OR MAGIC? THE PRESENTATION OF MAURICE MARTENOT \\ MUSICAL WAVES IN SPAIN
}

José Vicente Gil Noé

Universitat de València

\begin{abstract}
Resumen
En 1928, el músico francés Maurice Martenot presentó un nuevo instrumento musical electrónico en la Ópera de París al que llamó ondas musicales. Siendo uno de los pocos que gozó de éxito y que atrajo para sí la atención de los compositores, Martenot puso todo el empeño en su difusión ya desde una gira inicial que le llevó por todo el mundo entre 1931 y 1932. A partir de la información brindada por la prensa -pues no existen más referencias- este artículo pretende reconstruir la presentación del instrumento en España a lo largo de 1932, dar cuenta de la recepción por parte de la crítica y justificar cierto halo de misterio que lo envolvió tanto por su desconocido funcionamiento electrónico como por su forma de interpretarse solo con el movimiento de las manos en el espacio.
\end{abstract}

\section{Palabras clave}

Instrumentos musicales, electrónica, ondas, Martenot, éter, Andrés Gaos, crítica musical.

\section{Introducción}

En las primeras décadas del siglo $\mathrm{XX}$ tuvo lugar una verdadera revolución en el seno de la tecnología musical de la mano de la electrónica, una incipiente disciplina que, a la postre, acabaría dominando el siglo inundando todos los ámbitos de la vida. Si bien se pueden remontar a la segunda mitad del siglo XIX los primeros experimentos en la producción y transmisión eléctrica de sonido -los trabajos que apuntaban hacia una futura telefonía de Philip Reis o de Elisha Gray con su telégrafo musical (1874)- los primeros

\begin{abstract}
On 1928, the French musician Maurice Martenot presented at Paris Opera a new electronic musical instrument which he called musical waves. It had success like only a few and attracted the interest of the composers, Martenot strove to spread it with a initial tour that took it all around the world between 1931 and 1932. From the information given by the press -because there are not more references- this article aims to rebuild the presentation of the instrument in Spain all along 1932, to report about the reception by the review and to justify the mystery that involved the instrument because of its unknown electronic running and its performance only with the hands movement on the air.
\end{abstract}

\section{Key Words}

Musical instruments, electronics, waves, Martenot, ether, Andrés Gaos, music criticism

instrumentos musicales electrónicos ${ }^{1}$ no llegarían hasta los primeros años del siglo siguiente. ${ }^{2}$ El telharmonium (18971906) de Thaddeus Cahill o el choralcelo (1909) inventa-

1 RANDEL (2001): 385. Si bien las clasificaciones organológicas engloban bajo el nombre de instrumentos electrónicos diversos tipos, a lo largo del texto utilizaremos esta denominación para referirnos solo a aquellos instrumentos en los que el sonido se genera y se produce enteramente por medios electrónicos, dejando a un lado aquellos en los que el sonido se genera por medios mecánicos y se modifica electrónicamente.

2 HOLMES (2008): 6. 
do por Melvin Severy son solo dos ejemplos de aparatos ya electrónicos capaces de generar sonido a partir de las más diversas técnicas. Pero habría que esperar hasta la invención de la válvula de vacío por Lee De Forest entre 1906-1915 -componente electrónico clave en los circuitos osciladores que se empezarían a usar a partir de entonces- para que se hiciera efectivo el desarrollo de instrumentos electrónicos compactos y relativamente prácticos capaces de producir amplias gamas de sonidos enteramente por medios electrónicos. ${ }^{3}$

De entre todos los aparecidos en el primer tercio de siglo, desde el audion Piano del mismo Forest (1915), el theremin de Lev S. Termen (1917), el spherophone de Jörg Mager (1921) hasta el trautonium de Friedercih Trautwein (1930) por citar solo algunos, fueron las ondas musicales del francés Maurice Martenot (1928) las que mayor suerte corrieron en su difusión y aceptación por parte de los compositores y el entorno musical. ${ }^{4}$ Este instrumento, que contó con el potente apoyo de Francia, que se llegó a fabricar en serie, al que compositores de todo el mundo dedicaron más de mil obras, que ha gozado de cátedras en conservatorios y que ha contado con verdaderos virtuosos en su interpretación, se presentó en público en 1928 y protagonizó hasta 1932 una gira-presentación por países de todo el mundo que también incluyó a España.

Pero la producción electrónica de sonido -animada por aquellos que, como Ferrucio Busoni, los futuristas o Edgard Varèse, ${ }^{5}$ veían en las posibilidades tecnológicas un horizonte prometedor- no pasó, a la postre, de la imitación de los instrumentos tradicionales y de un tímido enriquecimiento de la paleta tímbrica orquestal. ${ }^{6}$ Como criticaba John Cage, el tratamiento de instrumentos como el theremin o las mismas ondas musicales de Martenot no hacía sino desperdiciar ingenuamente sus facultades, sin proporcionar nuevas experiencias sonoras explotando positivamente su capacidad para producir sonidos de cualquier frecuencia, amplitud o duración. ${ }^{7}$ Nuevas experiencias y nuevos comportamientos que llegarían ya con los nuevos medios y procedimientos

3 MARCO (2002): 292. La válvula de vacío tuvo un papel verdaderamente importante para la música del siglo XX, no solo por la creación de instrumentos electrónicos. De su mano vino el desarrollo de la radio, tan importante para la difusión musical, y en el futuro haría posible el nacimiento de la electroacústica.

4 ARACIL (1984): 54-55.

5 COX y WARNER (2008): 21. Como se recoge en diversas citas, Varése reclamaba nuevos instrumentos que pudieran enriquecer el alfabeto musical y no dudaba en proponer el trabajo conjunto de compositores y electrónicos.

6 LANZA (1986): 116.

7 CAGE (2007): 3. Charla pronunciada en Seattle en 1937 luego publicada como su célebre "credo". electroacústicos que pondrían en tela de juicio el temperamento igual y los esquemas tradicionales del discurso musical.

Así, más que sus entresijos técnicos o las posibles influencias estilísticas de un instrumento que en España ha tenido poca o nula trascendencia, se plantea en este caso un acercamiento a la recepción que tuvieron las ondas musicales de Martenot en las diversas ciudades españolas donde se presentaron. A partir del estudio del seguimiento que hizo la prensa de tal acontecimiento, este artículo tiene por objeto reconstruir la pequeña tournée que Maurice Martenot realizó por el país a lo largo de 1932. Estando por entonces España poco o nada acostumbrada a sobresaltos técnicos y novedades en general en el ámbito musical, las críticas y columnas en diarios configuran una rica y clarificadora visión de cómo se recibió y valoró el novedoso instrumento y, de forma más general, cómo se estimaba la aplicación de la electrónica al ámbito musical.

Precisamente las incógnitas que la electrónica encerraba todavía para la mayoría y el peculiar modo de interpretar el instrumento a distancia o sobre el teclado pero sin pulsar tecla alguna, solo con el movimiento libre de la mano en el espacio, acabó por dotar a las ondas de cierto componente de misterio. La prensa contribuyó a que su sonido y funcionamiento electrónico se inundaran de mística musical llegando a comparar a Martenot con un brujo cuyos procedimientos bien se podían considerar entre la música y la magia.

\section{Monsieur Martenot y sus ondas musicales}

Maurice Martenot (1898-1980) fue un violonchelista francés, verdadero apasionado de la ciencia y la tecnología, campos en los que se formó de manera autodidacta. Especialmente interesado por los problemas educativos, investigó acerca de la relajación y los estados físicos y psíquicos del individuo elaborando teorías y fórmulas pedagógicas junto a sus hermanos que acabarían por cristalizar en el conocido método Martenot para la enseñanza musical. ${ }^{8}$ Durante la Primera Guerra Mundial trabajó como telegrafista y operador de radio para el ejército, experiencias que le llevaron a investigar acerca de la generación electrónica de sonidos ya desde 1919. ${ }^{9}$

El músico francés trabajó durante tiempo en su instrumento y finalmente estableció la patente el 02.04 .1928 bajo el título de "Perfectionnements aux instruments de musique

8 ARNAUS (2007): 55-62.

9 BLOCH (2004): s.p. 
électriques" ${ }^{10}$ El 03.05.1928, solo un año después de que Lev S. Termen diera a conocer en la Ópera de París el theremin, Martenot presentó ante el público sus ondas musicales en el mismo escenario, interpretando como solista una obra encargada especialmente para la ocasión al compositor Dimitri Levidis (1886-1951): el Poème symphonique para ondas musicales solo y orquesta.

Consciente de que el éxito de un instrumento musical se halla en relación directa con el interés que despierta entre los compositores que pueden escribir para él, Martenot se esforzó en presentar y dar a conocer sus ondas musicales más allá de Francia y en suscitar la composición de obras que contaran con ellas. Tras el éxito de París, las ondas llegaron a Estados Unidos de la mano del director Leopold Stokowski que presentó la obra de Levidis dirigiendo a la Philadelphia Orchestra y al inventor en la Phliladelphia Academy of $\mathrm{Mu}-$ sic en diciembre de 1930. ${ }^{11}$ Entre 1931 y 1932 emprendió una tournée que le llevó por diversas ciudades de todo el mundo.

Un objetivo fundamental para Martenot fue perfeccionar el instrumento de forma que fuera versátil e inmediatamente familiar a músicos y compositores para asegurarse de que fuera ampliamente aceptado. Muy similar al theremin, consiguió darle una apariencia más musical -aquél era más similar a una radio que a un instrumento- y además logró facilitar su manejo y dotarlo de cualidades más expresivas. Parece que logró su cometido pues, a la postre, sus ondas musicales han sido el instrumento electrónico que más éxito ha tenido. Desde su presentación, más de mil trabajos comprenden su repertorio en los más variados géneros, desde obras sinfónicas hasta bandas sonoras, pasando por ballets, musicales, pop, rock e incluso anuncios. ${ }^{12}$ Además, el instrumento fue objeto de un programa formal de aprendizaje y el mismo inventor enseñó su interpretación en el Conservatoire National Supérieur de Musique de París desde 1947. Incluso han destacado grandes intérpretes además del propio Maurice Martenot, como su hermana Ginette Martenot, Jeanne Loriod -formada con el inventor-o la alumna de esta Valérie Hartmann-Claverie. ${ }^{13}$

El fundamento electrónico que seguían las ondas musicales para generar sonido estaba basado en el principio heterodino, el mismo que se usaba para la sintonización

10 BRICE (2012): 105.

11 HOLMES (2008): 25.

12 BLOCH (2004). Entre la larga lista de compositors que han escrito para Ondas Musicales se puede citar a Olivier Messiaen, Pierre Boulez, Sylvano Bussotti, Arthur Honegger, Jacques Charpentier, Darius Milhaud, André Jolivet o Jacques Ibert.

13 HOLMES (2002): 69. de la radio y con el que también se explicaba el funcionamiento de sus antecedentes más directos como el theremin o el spherophone..$^{14}$ Según este principio, el aparato contaba con dos osciladores -circuitos eléctricos formados por una fuente, un condensador y una bobina capaces de producir y mantener una oscilación de frecuencia determinada- que incorporaban válvulas tríodo y que diferían en frecuencia de manera sostenida. Las altas frecuencias producidas por ambos osciladores eran inaudibles pero la interferencia por síntesis sustractiva - controlada y calculada entre ambas acababa por producir una frecuencia situada dentro del régimen perceptible por el oído humano. Uno de los osciladores -el fijo- permanecía en una frecuencia constante, sin modificación alguna a lo largo del manejo del aparato, mientras el otro -el variable- alteraba su frecuencia por la acción indirecta del intérprete -que con sus movimientos o pulsaciones provocaba un cambio en la capacitancia del condensador- obteniéndose de la diferencia entre ambas frecuencias las diversas resultantes audibles, es decir, generándose los diferentes sonidos.

Si bien se regían por el mismo principio, la forma en que el intérprete conseguía modificar la frecuencia del oscilador variable y así cambiar de un sonido a otro, era diferente entre los distintos instrumentos. Así, mientras en el theremin no había contacto físico y el efecto se conseguía por el movimiento de la mano respecto a una antena, en las ondas musicales de Martenot siempre hubo contacto físico -aunque ligero- entre intérprete e instrumento. ${ }^{15}$ Una forma de contacto, de manejo, que fue variando a lo largo del tiempo, pues desde su creación se sucedieron hasta siete variantes de las ondas musicales, cada una siempre con alguna innovación respecto a la anterior. ${ }^{16}$ En el modelo presentado en 1928, Martenot se situaba a un par de metros de distancia del cuerpo del aparato y lo manejaba tirando o aflojando de un cable -fijado a su mano derecha a través de un anillo- que actuaba sobre una polea en el instrumento alterando la frecuencia del oscilador variable y, como resultado de ello, el sonido generado. Con una posterior modificación, Martenot añadió dos formas más de manejo o interpretación: un teclado similar al del piano, pero con un añadido movimiento lateral para lograr el efecto de vibrato; y una cinta justo por delante del teclado, con un anillo en el que introducir el dedo índice de la mano derecha, que permitía obtener diferentes sonidos y glissandi con un movimiento lateral continuo, teniendo como referencia visual para la afinación las teclas y una banda con muescas y protuberancias. Este fue

14 SCHOLES (1984): 693

15 GLINSKI (2000): 126.

$16<\mathrm{http}: / /$ www.thomasbloch.net/en_ondes-martenot.html>. (Consulta marzo 2013). 
precisamente el modelo que llegó a España en 1932, con sus tres formas posibles de interpretación. ${ }^{17}$ Con posterioridad, el instrumento perdería la posibilidad de manipularse a distancia y solo conservaría el teclado y la cinta frente a este como formas de variar los sonidos.

El instrumento de Martenot es monofónico y en los casos comentados, incluido el presentado en España, el intérprete solo utiliza la mano derecha para cambiar de un sonido a otro. Con la mano izquierda se maneja un cajón o panel de control con botones, interruptores y pastillas que permiten variar el timbre, controlar la envolvente acústica -para disponer ataques y disoluciones-, determinar las intensidades o controlar los difusores.

El formato físico de las ondas musicales ha variado poco desde el que se viera y escuchara en España en 1932. Consta de una unidad principal, donde está el teclado, el cajón de mandos para la mano izquierda e, interiormente, toda la electrónica. Además consta de tres difusores. El principal es un altavoz convencional; un segundo difusor llamado palma es un cuerpo de resonancia con forma de llama y un agujero, que consta de doce cuerdas en sus partes anterior y posterior que vibran por simpatía; un último difusor llamado metálico es una pieza con forma de altavoz cuyo espacio para el diafragma lo ocupa una pieza metálica suspendida a modo de gong que añade un halo metálico al sonido.

\section{Tournée por España: crónica de una presentación}

Entre los países que Maurice Martenot visitó en los primeros años treinta, en lo que fue una primera gira para mostrar y dar a conocer su instrumento como paradigma del potencial musical de la electrónica, se encontraba España. El músico francés visitó Madrid y Barcelona en la primavera de 1932, ofreciendo varias audiciones en ambas ciudades; poco después, y ya en concierto único, se presentó en La Coruña durante la misma primavera y en Valencia, Alicante y Vigo a lo largo del otoño del mismo año. Llegó en compañía de su hermana Ginette Martenot, como venía siendo habitual, y contó en todos los conciertos con la colaboración del violinista y compositor gallego Andrés Gaos para acompañar sus interpretaciones con el piano. ${ }^{18}$ Martenot tuvo en muy buena

17 El Debate (06.04.1932): 3. Así hacía referencia Joaquín Turina a los tres modos de interpretación: "Para captar este sonido emplea el señor Martenot tres modos de actuar: uno de ellos, el más aparatoso, consiste en una planchita unida por un hilo al aparato; la segunda manera es una cinta que gira sobre el teclado, sin tocarlo; el tercer modo es tocando el teclado como en un piano".

18 Diario de Valencia, (13.11.1932): 2. "En su actual excursión por España acompaña a Martenot un artista [...] Andrés Gaos que, ahora, con exquisito tacto colabora como pianista acompañante al éxito de consideración la colaboración del músico español que, además del estricto acompañamiento musical, ayudó explicando al auditorio la base científica y musical de las ondas. ${ }^{19}$

Si bien los conciertos de Valencia, Alicante y Vigo se celebraron en teatros -los Teatro Principales y el Teatro García Barbón respectivamente, por aquella época los mejores espacios musicales posibles en esas ciudades- en el caso de las audiciones de Madrid y Barcelona Maurice Martenot tuvo que conformarse con sendos cines para su presentación. Ello resultaba sorprendente siendo las dos ciudades más importantes del país y, sobre todo, contrastaba con la categoría de los escenarios por los que había pasado el instrumento del músico francés desde su aparición en 1928 en la mismísima Ópera de París. ${ }^{20}$ Martenot no pudo sino lamentar esta situación que, quizá, alimentó una consideración de su trabajo más como atracción o curiosidad científica que como verdadero acontecimiento musical, algo que ya de por sí venía dado, en parte, por la curiosa forma de manejar el instrumento. ${ }^{21}$

Teniendo en cuenta la edad todavía temprana del instrumento -presentado en público apenas cuatro años antes y todavía no conocido ampliamente-, y aunque ya existía alguna obra expresamente escrita él -sin ir más lejos el Poème symphonique de Levidis-, el programa no tenía novedad alguna y estuvo íntegramente compuesto por fragmentos de obras célebres del repertorio: ${ }^{22}$

Martenot El mismo señor Gaos dio lectura, por la dificultad de explicación de lo que es el instrumento de la música de las ondas aéreas [...]". A un lado esta faceta de pianista, Andrés Gaos (1874-1959) destacó como violinista de reconocimiento internacional y como compositor, con una obra de carácter romántico, con algún acercamiento al expresionismo y un trasfondo nacionalista. Cfr. MARCO (1983): 99.

19 "Gaos me acompañaba a lo largo de todo el programa, su musicalidad era una preciosa colaboración a mis conciertos, donde la delicadeza de ciertas sonoridades del instrumento de ondas exigía del acompañante una calidad de toque y una fineza de interpretación que pocos pianistas pueden obtener". Texto atribuido a M. Martenot. <http://www. andresgaos.com/spanish/fotograndes/1932.htm> (Consulta marzo 2013).

20 Entre otros, la Philadelphia Academy of Music, el Teatro Imperial de Viena o el Teatro alla Scala de Milán.

21 La Época (12.4.1932): 4. "Lamentaba, con razón, este artista y hombre de ciencia de no haber recabado para sus actuaciones los auditorios habituales de las sesiones propiamente musicales y haber sido, en cambio, considerado, en general, como una, atracción, casi como una de esas curiosidades científicas que, de cuando en cuando, ennoblecen los programas de una función de variedades. Quizá no ha sido ofrecido este curiosísimo espectáculo francamente artístico, en el medio y la oportunidad indispensables".

22 La lista de obras aparecía en el programa de mano publicado por la Sociedad Filarmónica de Vigo: $C f r$. <http://www.andresgaos.com/ spanish/fotograndes/1932.htm> (Consulta marzo 2013); así como en el publicado por la Sociedad Filarmónica de Valencia: Cfr. SAPENA (2007): 988-989; y coinciden con el esbozado en la prensa madrileña: La Libertad (08.04.1932): 8; y barcelonesa: (La Vanguardia (12.04.1932): 23. 


\section{Primera parte}

Coral. BACH

Melodía. GLUCK

Canto hebreo. PAUER

Cantos rusos. LALO

Segunda parte

Largo. HAENDEL

Dos valses. BRAHMS (transcripción para ondas por Ginette Martenot)

Canto indio. RIMSKI-KORSAKOV

Minuetto. BEETHOVEN

Danza oriental. RIMSKI-KORSAKOV

Tercera parte

Suite. CAIX D'HERVELOIS

Vals (El canario). MOZART

Canción de Solveig. GRIEG

La mañana. GRIEG

Centelleo. SCHMITT-GINETTE MARTENOT

Melodías populares. ANÓNIMO (transcripción para ondas por Ginette Martenot).

La crítica señaló en alguna ocasión la falta de interés musical de las piezas, ${ }^{23}$ pero lo cierto es que se trataba más de una demostración de posibilidades que de un concierto al uso, siendo las obras elegidas lo que menos importaba del evento. De hecho, en algunos casos se subrayaba el componente científico: "¿Ciencia o arte? He aquí el misterio que el porvenir se encargará de resolver", se preguntaba Joaquín Turina; ${ }^{24}$ en otros, la prensa presentaba el acontecimiento en términos más propios de la experimentación científica que de la exhibición musical: "Más que una demostración artística se celebró ayer una ejemplificación de cátedra de Física [...] El público de la Filarmónica quedó ayer interesado por el curioso experimento [...]". ${ }^{25}$

Maurice Martenot empezó su periplo en tierras españolas por la capital. El día cinco de abril tuvo lugar la presentación en el Cine Rialto, una audición privada para la que convocó a críticos musicales, periodistas, compositores y músicos. ${ }^{26}$ Tras esta primera aparición se programaron dos conciertos más en el mismo espacio los días siete y ocho del mismo mes y todavía, a petición del público, ${ }^{27}$ se rea-

23 La Correspondencia de Valencia (15.11.1932): 3. "El programa, seguramente confeccionado ex profeso, no ofrecía nada de interés, pues todo él era sobradamente conocido [...]".

24 El Debate, (06.04.1932): 3.

25 Las Provincias (13.11.1932): 1-2.

26 El Heraldo de Madrid (05.04.1932): 14; La Época (05.04.1932): 4.

27 La Libertad (09.04.1932): 8. El anuncio en el diario de este lizó uno último el día nueve que no daba sino cuenta de la buena acogida entre los madrileños y de la expectación suscitada..$^{28}$

El seguimiento por parte de la prensa fue masivo y, así, encontramos que entre los días seis y doce de abril los diarios más importantes de Madrid -como $A B C, E l$ Debate, El Heraldo de Madrid, El Sol, La Época, La Libertad o La Voz, así como una recién nacida revista Ritmo- dieron cobertura a la presentación de las ondas musicales de Martenot con sucesivos anuncios y críticas de los conciertos.

La publicidad aparecida los días previos al evento, buscando crear la mayor expectativa posible, seguía la línea de aquella que, desde algunos años atrás, venía anunciando los conciertos de Theremin con su instrumento, ${ }^{29}$ haciendo especial hincapié en el protagonismo del éter y en la producción de música únicamente con el movimiento de las manos en el espacio. Algunos de aquellos anuncios llamaban así la atención del lector de prensa:

"Martenot ha demostrado en el Teatro de la Ópera de París, que se puede captar la música del aire sin necesidad de instrumentos". 30

“CCómo es posible que sin instrumentos puedan ejecutar obras musicales? Martenot os lo demostrará en Rialto. Mañana jueves noche, en su primer concierto. Es algo nuevo, original, asombroso, que jamás se ha escuchado en nuestro planeta. No se trata de música artificial, sino absolutamente natural, captada del éter". ${ }^{31}$

"Martenot, el hombre que con el sólo movimiento de las manos en el espacio ejecuta obras musicales se presentará muy pronto en Madrid en dos únicos conciertos. Absolutamente nadie debe dejar de asistir a escuchar la música de las ondas sonoras en...". ${ }^{32}$

último concierto rezaba: "Hoy sábado a las seis treinta, a petición del público, Martenot dará su último concierto de la música de las Ondas Aéreas".

28 La prensa recogía el interés demostrado por el auditorio: $E l$ Heraldo de Madrid (05.04.1932): 14: "El numeroso auditorio quedó realmente asombrado y tributó entusiastas ovaciones al inventor". $E l$ Debate (06.04.1932): 3: "[...] ovacionaba a Martenot y trataba de comprender y descifrar el extraño aparato". La Libertad (08.04.1932): 8: "El público aplaudió cordialmente todos los números y salió complacidísimo del interesantísimo concierto".

29 Por ejemplo el cartel que anunciaba la primera demostración de Theremin en Estados Unidos en 1928. Sobre un dibujo de fondo en el que se veían dos manos rodeadas de múltiples líneas sinuosas que copaban el espacio gráfico, decía: "Music from the ether. Produced by free movement of hands in air".

30 El Heraldo de Madrid (02.04.1932): 5.

31 El Sol (06.04.1932): 2.

32 La Época (04.04.1932): 5. 


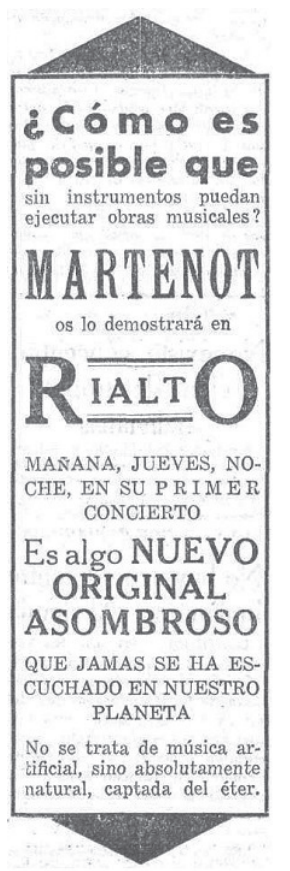

Figura 1. Anuncio de concierto en Madrid. El Sol (06.04.1932), p.2

Las críticas y las columnas de los diarios se centraron en analizar y dar cuenta de las características técnicas y musicales de tan atípico y misterioso instrumento. En los intentos por explicar su funcionamiento, se abordaba el principio electrónico y se aludía al éter y su papel como emisor y propagador de las vibraciones para la explicación básica del aparato:

"Pero el aparato de Martenot está fundado en un novísimo principio, en el que no juega papel alguno la materia, al menos en los estados físicos reconocidos hasta hoy, dado que el agente emisor de las vibraciones y propagador de ellas es el éter de los físicos [...] en este novísimo aparato que comentamos se origina el fenómeno en el interior de los tubos termoiónicos, o de vacío, y por un fenómeno análogo al del teléfono, las vibraciones transmitidas por los hilos de la instalación radiotelefónica son traducidas por el diafragma del altavoz en sonidos musicales. Es decir, que la causa original no es un sonido (como ocurre en el teléfono o el gramófono): la causa original emisora es, o son, variaciones de oscilación en la emisión electrónica del tubo [...]".33

33 La Libertad (06.04.1932): 6.
Las valoraciones en los diarios subrayaban el hecho, entonces sorprendente, de que para producir el sonido no se necesitaran los habituales medios mecánicos: "Es un aparato sin cuerdas ni martillos ni accesorio alguno que pueda producir sonido", explicaba un colaborador de Ritmo. ${ }^{34}$ Animados por la curiosidad del novedoso instrumento, fueron muchos -como relataba Joaquín Turina ${ }^{35}$ - los que se acercaron en los intermedios para examinar de cerca las ondas musicales y comprobar cómo en su interior solo se hallaban válvulas y demás elementos eléctricos que hacían pensar más en un aparato de radio que en un instrumento musical. Cualquiera podía convencerse de que aquello que admiraban, pese a no contar con los elementos tradicionalmente creadores de vibraciones musicales, producía sonido por sí mismo, haciendo nacer las ondas sonoras directamente de la electricidad:

"Electricidad, lámparas, varios metros, mandos... No... no se trata de la radio. No es el instrumento creado por Martenot un «reproductor» de sonidos, sino un «productor», un creador de sonidos, de tal manera, que en él desaparecen aún los elementos insustituibles de la producción sonora musical (cuerdas, arcos, boquillas, lengüetas...) quedando sólo la vibración misma, la vibración sola, lo que podríamos llamar el sonido cimero y manantial". ${ }^{36}$

Fue especialmente comentada la respuesta del instrumento simplemente a los movimientos del intérprete con la mano: "Por el sólo movimiento de la mano en el espacio se ejecutan obras". ${ }^{37} \mathrm{La}$ variante del instrumento de las ondas musicales que Martenot presentó en España mantenía, además del teclado y la cinta horizontal con anillo frente a este, la fórmula original para producir sonido con el movimiento de una cinta que el intérprete -situado a uno o dos metros del mueble- unía a su mano a través de un anillo y movía a voluntad. Joaquín Turina explicaba: "extraño aparato, que parecía un sencillo pianito pero que sonaba sin necesidad de pulsar sus teclas". ${ }^{38}$

Entre las cualidades puramente musicales del invento de Martenot llamó la atención de los sectores especializados la limpidez de su sonido y su versatilidad tímbrica. En tanto que la producción del sonido no partía de elementos materiales o accionamientos mecánicos, en las ondas la crítica

34 Ritmo, 54 (abril de 1932): 14.

35 El Debate (06.04.1932): 3. “[...] no niego que, en compañía de otros curiosos, he asaltado el escenario para contemplar de cerca el admirable instrumento misterioso".

36 La Época (12.04.1932): 4.

37 El Sol (07.04.1932): 5.

38 El Debate (06.04.1932): 3. 
apreció sonidos "de una belleza y acuidad, de una pureza tan limpia y aérea que da placer singular" ${ }^{39}$ Y ello gracias a un modo de emisión que no arrastraba imperfección o lastre mecánico alguno:

"Así pues tenemos aquí un modo de emisión que supera en agilidad a todos los medios mecánicos y materiales que pudiéramos inventar, y de una pureza inconcebible, por cuanto ninguna causa, tosca o material, viene a sumarse a la vibración del éter. Y a esto precisamente se debe la belleza de los sonidos que tanto cautivó al público y a los críticos presentes en la experiencia de ayer. [...]. Considérese la ventaja de un modo tan sutil, tan inmaterial, que puede variar sin el menor esfuerzo, dentro de inmensos límites, sin temor a rascar, frotar o soplar más o menos, ni a escuchar el soplo, el frote, la percusión ni ninguno de los desagradables efectos que produce la sensación de materia que muchas veces dan los instrumentos. Es la vibración, la vibración pura, virgen de contacto material, lo que origina el sonido en este tipo de música. ¿A qué grado de belleza puede elevarse esta música, música en verdad etérea al menos en su origen?". 40

No pasó inadvertida la capacidad del instrumento para mudar su timbre gracias a los filtros y procedimientos electrónicos que el intérprete podía poner en juego desde el cajón que gobernaba su mano izquierda. Cada columnista señaló una retahíla de timbres fingidos que le pareció apreciar en las sucesivas transmutaciones de las ondas, desde la emulación del violín, el chelo, el oboe o la flauta hasta el cuerno de caza o la guitarra hawaiana. ${ }^{41}$ Un efecto definido en algún caso como de desconcertante "ventriloquía". ${ }^{42}$

De la manipulación del aparato sin contar con el teclado - a través del anillo o de la cinta a distancia- no solo se obtenía un llamativo y relumbrante efecto de glissandi. A nadie se le escapó la posibilidad de detenerse en frecuencias cualesquiera más allá de las doce de un temperamento igual que, por entonces, todavía se mostraba hegemónico, cuestionado solo por algunas ya célebres tentativas extranjeras - Busoni, Carrillo, Haba o Wyschnegradsky entre otros- e incluso por algunas nacionales tristemente poco conocidas como las de Juan Domínguez Berrueta, Eduardo Panach Ramos e incluso Juan García Castillejo. ${ }^{43}$ Es cierto que las teclas de

39 La Libertad (06.04.1932): 6.

40 La Libertad (06.04.1932): 6.

41 El Heraldo de Madrid (05.04.1932): 14.

42 La Época (12.04.1932): 4.

43 GIL NOÉ (2013): 99-105. Domínguez Berrueta trabajó en la "regeneración de la gama de los sonidos" desde un plano más teórico y García Castillejo apuntó hacia el microtonalismo como una de las las ondas musicales de Martenot tenían cierta holgura hacia los lados para permitir una ligera variación de la afinación, pero su objeto era más expresivo que acústico, en busca de un efecto de vibrato que animara el sonido puro, plano. Pero obviamente, con las otras dos formas de obtener sonidos con el movimiento, todas las fracciones acústicamente posibles estaban, nunca mejor dicho, al alcance de la mano:

"Si se consigue que el aparato, por medio de su actuación eléctrica, produzca sucesivamente el número de vibraciones exactas en que se coagula cada una de las notas de las escalas musicales, se hallará en condiciones de interpretar toda suerte de melodías. Su posibilidad de detenerse ante cualquier número de vibraciones lo capacita no sólo para hacerse intérprete de las escalas de nuestro sistema musical, sino de las de otro cualquiera que no guarde la misma relación matemática. Pues todos los lectores medianamente ilustrados no ignoran que los doce escalones en semitonos en que dividimos nosotros la gama pueden ser ampliados y también disminuidos". ${ }^{44}$

La demostración de Maurice Martenot permitió a los asistentes a los conciertos comprobar un registro amplísimo, una posibilidad de variación en altura de enormes posibilidades y sorprendentes efectos. Ante los recorridos del agudo al grave y viceversa mostrados por el intérprete, el auditorio pudo apreciar desde frecuencias tan graves que permitían contar las vibraciones hasta otras tan agudas que se perdían en lo ultrasónico, inaprehensibles para el oído humano. ${ }^{45}$

Entre tantas consideraciones positivas aún hubo espacio para algún reproche. Joaquín Turina echaba de menos, siendo como era el instrumento monofónico, la polifonía de voces para poder obtener resultados verdaderamente prácti$\cos { }^{46}$ Por su parte, Mateo H. Barroso no es que cuestionara la inapelable necesidad de contar con altavoces para amplificar el sonido, pero en un tono ciertamente utópico y hasta místico imaginaba la posibilidad de poder escuchar, ante un instrumento como el de Martenot, el sonido en su estado más puro e inmediato:

grandes posibilidades de su "música eléctrica". Fue quizá el valenciano Eduardo Panach quien abordó el microtonalismo de forma más global: sistematizó un sistema de tercios de tono y otro que dividía la octava en 53 partes, pero no solo investigo y empezó a publicar sus resultados sino que además creó dos instrumentos microtonales -una triola y una guitarra afinada en semitonos, cuartos y tercios de tono- y compuso varios boceto sinfónicos aplicando de forma práctica sus sistemas. $44 \mathrm{La} \mathrm{Voz}(06.04 .1932): 3$.

45 El Debate (06.04.1932): 3. Joaquín Turina explicaba haber sido testigo de "[...] sonidos tan graves que se podían contar las vibraciones y otros, agudísimos, que el oído no percibía por su altura [...]". 46 El Debate (06.04.1932): 3. 


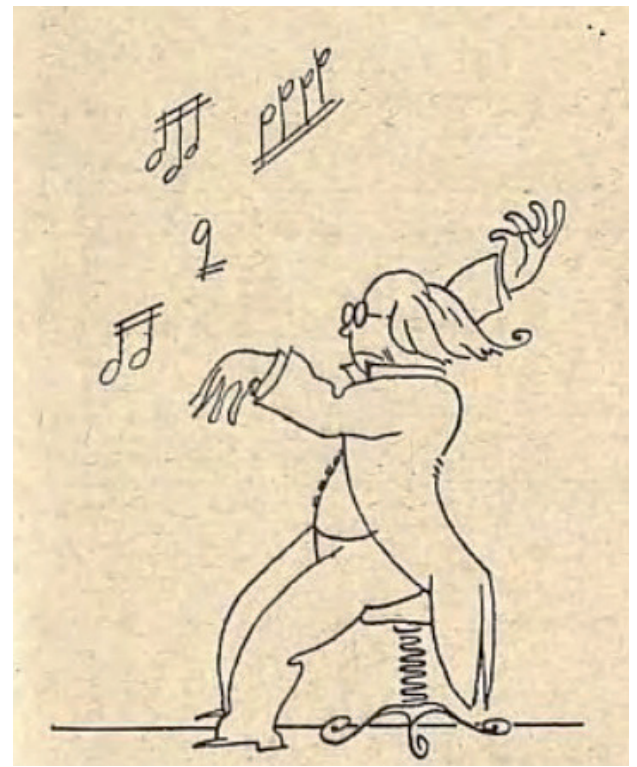

Figura 2. Caricatura de un "músico imaginario" captando ondas del éter. Semanario barcelonés Popular Film $n^{\circ} 296$ (14.04.1932), p. 7

"Y no es aún el sonido puro; todavía precisa el altavoz, o el diafragma vibrante, que traduce en sonido las vibraciones del éter. ¿Llegaremos a oír el sonido directamente sin intermedio material, tal como debe ser la vibración pura, limpia y virginal del éter? Posiblemente no es dado al hombre el conseguirlo. Ello equivaldría a percibir directamente la armonía de los mundos y la melodía de las esferas". ${ }^{47}$

Tras el primer contacto de monsieur Martenot y su instrumento con el público y el entorno musical español en Madrid, el francés partió hacia Barcelona donde daría continuidad a su gira con tres conciertos más. El programa era el mismo que había presentado en la capital y, como allí, a falta de un escenario mejor se presentaba en un cine, en este caso el Cine Coliseum. Un primer concierto tuvo lugar el 12.04.1932, privado, bajo rigurosa invitación y en primicia para autoridades, críticos, prensa, compositores, intérpretes, técnicos de radio y cine sonoro, hombres de ciencia -químicos, físicos- y personalidades del mundo artístico, literario y musical ${ }^{48} \mathrm{Al}$ mismo siguieron dos conciertos públicos los días trece y quince del mismo mes, anunciados en prensa con anterioridad con la misma voluntad de levantar la cu-

47 La Libertad (06.04.1932): 6.

48 La Vanguardia (10.04.1932): 15; (12.04.1932): 23. riosidad del lector y crear la máxima expectativa. Además de los anuncios, los días previos a los conciertos la prensa realizó un seguimiento de las audiciones madrileñas para poner en antecedentes al público local, destacando los mismos aspectos de las ondas musicales que subrayaban los medios de la capital. ${ }^{49}$

El tono de humor lo ponía el semanario cinematográfico barcelonés Popular Film. Ante las especulaciones con el éter, el "misterioso" funcionamiento del instrumento y su forma asombrosa de interpretarse con el movimiento de las manos, junto a una caricatura comentaba:

"Hasta ahora el éter servía, entre otras cosas, para hacerlo aspirar por las señoras que se desmayaban oportunamente, pero Maurice Martenot lo ha convertido en instrumento musical, un instrumento, desde luego, que no se parece a ninguno conocido, ni siquiera al acordeón. [...] Hay para pensar si no será Martenot un músico imaginario, o si se trata del espíritu de Beethoven". ${ }^{50}$

Con posterioridad a las tres actuaciones en Barcelona, Maurice Martenot todavía visitó otras ciudades españolas en conciertos únicos a lo largo de 1932. Sin descartar que pudiera presentarse en otras capitales de las que hasta el momento no tenemos noticia, el músico francés mostró las ondas musicales en La Coruña el veintiséis de abril,$^{51}$ en el Teatro Principal de Alicante el dieciocho de noviembre, ${ }^{52}$ y en el Teatro García Barbón de Vigo el seis de diciembre. ${ }^{53}$

Entre las citadas, aún hubo otra audición de Martenot en el Teatro Principal de Valencia el doce de noviembre, organizada por la Sociedad Filarmónica de la ciudad para el concierto número 181 de la temporada 1932-1933.54 Los diarios más importantes de la ciudad dieron cuenta del acontecimiento y los críticos y columnistas resaltaron los aspectos más llamativos del instrumento: su capacidad para aprovechar las ondas etéreas y convertirlas en sonido para la interpretación de música a voluntad, las diversas formas de interpretación, el amplio registro y la variabilidad tímbrica. ${ }^{55}$ Pero lo más destacable de la valoración de la prensa fue la crítica acerada de V. Llopis Piquer en el diario local El Pue-

49 La Vanguardia (05-15.04.1932).

50 Popular Film (14.04.1932): 7.

51 La Vanguardia (26.04.1932): 20.

52 La Vanguardia (19.11.1932): 21.

53 Concierto número 222 organizado por la Sociedad Filarmónica de Vigo.

54 SAPENA (2007): 988-989.

55 Diario de Valencia (13.11.1932): 2; La Correspondencia de Valencia (15.11.1932): 3; La Voz Valenciana (14.11.1932): 1; Las Provincias (13.11.1932): 1-2. 
blo, arremetiendo duramente contra Martenot y queriendo desmentir dos puntos para él importantes: la supuesta novedad del instrumento y el parentesco con la radio:

“[...] si no es tomadura de pelo, puede considerarse como supina y crasa ignorancia, ya que no queremos pensar en la mala fe de estimar que el público valenciano es oriundo del Japón, de donde se dice acaba de llegar el señor Mauricio Martenot. [...] En primer término, Mauricio Martenot NO HA INVENTADO ABSOLUTAMENTE NADA fundamental con referencia a su aparato, como se aseguró en el curso de la experiencia repetidas veces, y vamos a demostrarlo: [...] el procedimiento es el mismo de Theremin: acercar o separar la mano da distintas notas. [...] Sin registro, pedal, teclado, bastando con un gesto, el profesor Theremin hace bastantes años realizaba ya el "prodigio" que pudimos contemplar en la Filarmónica [...]. Y que conste que lo único que el profesor Theremin no consiguió fue lo más brillante del concierto de Martenot. Los efectos de eco, maravillosos... ; colocando un altavoz en el salón de fumar del cuarto piso del teatro! [...]. ¿Cómo se realiza este fenómeno que no tiene nada tampoco de MISTERIOSO ni de DESCONOCIDO? [...] Y conste también que al hablar así desmentimos otra afirmación de las vertidas, o sea la de que el aparato expuesto en la Filarmónica nada tiene que ver con la radio, siendo así que esta ciencia es la base precisamente del mismo, que si trabaja es por las lámparas que lleva y las corrientes de alta frecuencia que las mismas producen". ${ }^{56}$

\section{Tocar "con el solo movimiento de las manos en el espacio". Entre la música y la magia}

A la descripción y valoración de las peculiaridades musicales de las ondas de Martenot, buena parte de la prensa añadió un relato de tintes mágicos que envolvía tanto al instrumento como a su creador en una suerte de halo enigmático e incluso místico, en tanto que los hacía protagonistas del misterio del "sonido puro" y poseedores de cierta razón oculta. Ello no solo respondía a una voluntad de provocar interés y expectativas, sino que daba cuenta de con cuántas incógnitas se vivían y entendían en el ámbito de la cotidianidad -más allá del círculo científico de la física al que la mayoría permanecía ajena- el todavía incipiente fenómeno electrónico y la presencia de un arcano elemento etéreo.

El fin que Martenot persiguió era en origen eminentemente práctico, dotar de nuevos medios al compositor y enriquecer con nuevos colores tímbricos la paleta orquestal

56 El Pueblo (15.11.1932): 3. gracias a las posibilidades de la electrónica. Pero pese a estar alejado en principio de ideas místicas -que sí encontraron sustento en otros pioneros del sonido electrónico como Jörg Mager $^{57}$ - no pudo evitar que sus ondas musicales se asociaran con una "peligrosa mística musical". ${ }^{58}$ Pesó en esa asociación la similitud con el theremin -que ya había padecido la vinculación con lo misterioso ${ }^{59}$ - y el hecho, innegablemente llamativo, de que el aparato respondiera con sonidos al simple movimiento de las manos en el espacio. Un espacio que se creía todavía dominado por el éter invisible -esa hipotética substancia o supuesto medio que todo lo ocupaba y que era sede de los fenómenos ópticos y electromagnéticos- a pesar de que su necesaria existencia había sido ya descartada por Alfred Einstein en su Teoría de la Relatividad de $1905 . .^{60}$

La ausencia en algunos instrumentos electrónicos de medios materiales que poner en vibración alimentaba la idea de que el movimiento de las manos del intérprete no hacía sino recoger, captar ondas etéreas que el aparato transformaba en sonidos. Así había ocurrido antes con el theremin cuyo inventor, de hecho, lo llamó inicialmente eterófono ${ }^{61}$ y ahora con las ondas musicales: “[...] una persona, sin más que mover un brazo en el espacio, capta ondas aéreas y produce sonoridades musicales, cual si fuese una orquesta la que tocase". ${ }^{62}$ No unos sonidos cualesquiera, aquella música no se presentaba convencional: "No se trata de música artificial, sino absolutamente natural, captada del éter", ${ }^{63}$ con una pureza tímbrica que, precisamente, se explicaba por su origen en el inmaterial elemento. ${ }^{64}$

57 PRIEBERG (1964): 278. Según el autor, Mager tenía “sueños" de una mística relación entre la vibración musical y el número significativo, y de un sonido electrónico que influyera de un modo curativo en el cuerpo y en el alma. El nombre de su instrumento, el spherophone, se relacionó con la música de las esferas pitagórica.

58 PRIEBERG (1964): 282.

59 GLINSKI (2000): 82. El autor recoge un comentario de prensa de la época que se refería al músico en estos términos: "A few hundreds of years ago Professor Theremin would have been condemned for performing black magic. In the minds of some music lovers today he still has to prove himself innocent".

60 GONZÁLEZ DE POSADA (2001): Tomo CXVIII-cuaderno 1, 43-72.

61 TARUSKIN (2010): 181. Del nombre inicial de eterófono pasó a termenvox tras el acuerdo en la patente firmado entre la URSS y Alemania en 1924. Posteriormente cambió su apellido y el nombre de su instrumento por el definitivo y más conocido de theremin.

62 ABC (06.04.1932): 35.

$63 \mathrm{El} \mathrm{Sol}(06.04 .1932): 2$.

64 La Libertad (06.04.1932): 6. “[...] un modo de emisión de una pureza inconcebible, por cuanto ninguna causa, tosca o material, viene a sumarse a la vibración del éter. Y a esto precisamente se debe la belleza de los sonidos [...] ¿A qué grado de belleza puede elevarse esta música, música en verdad etérea al menos en su origen?". 
Los anuncios que se sucedían en los diarios no vacilaban en presentar la experiencia de escuchar y ver en acción a Martenot y su instrumento más cerca de la ilusión que de la realidad: "Tan extraño, tan inconcebible es el ya famoso invento de Martenot. El músico mágico" ${ }^{65}$ Incluso hacían del músico francés un inesperado hechicero de los sonidos: "El brujo que con el sólo movimiento de las manos, en el espacio ejecuta obras musicales". ${ }^{66}$

No solo los anuncios mostraban este cariz, también las impresiones de algunos columnistas que llevaban la audición al terreno de la ficción: "Realmente, arrancar el sonido del éter y transmitirlo al público por medio de una combinación de pilas eléctricas parece un cuento de hadas"; $; 7$ y de otros que veían en Martenot un verdadero nigromante:

“Diríamos que la mano bruja de Martenot recorre en el espacio un mástil irreal, en busca de notas para un violoncelo fantástico? O bien que una sugestión suavemente imperativa impone vibraciones a los elementos imponderables, esclavos de la ciencia humana, señora, esta vez, del arte divino?". ${ }^{68}$

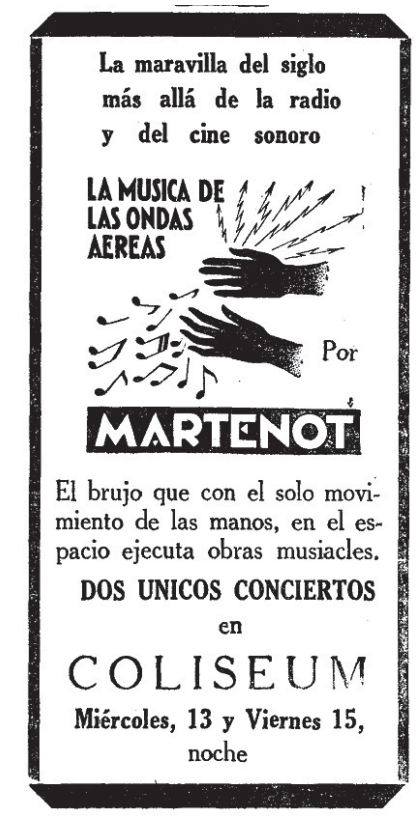

Figura 3. Anuncio de concierto en Barcelona. La Vanguardia (10.04.1932), p. 13

65 La Libertad (07.04.1932): 8.

66 La Vanguardia (10.04.1932): 13.

67 La Época (07.04.1932): 2.

68 La Época (12.04.1932): 4.
Incluso parece que las sensaciones y comentarios del público durante y tras el concierto, realmente impresionado como se desprende de todas las crónicas, alimentaba la comparación con la exhibición mágica: "El público que ayer escuchó en Rialto el aparato del seño Martenot, quedó sencillamente maravillado, estupefacto, como en una función de taumaturgia". ${ }^{99}$

Tanto anuncios como comentarios periodísticos en este tono estaban claramente influidos por una prensa extranjera que había dejado impresiones y titulares verdaderamente sugerentes a propósito de conciertos anteriores de las ondas Martenot por todo el mundo: "Hay algo de brujería en esta música de las ondas, que el aparato eléctrico de Martenot capta de la atmósfera" explicaba Le Figaro en París; "No hay vocabulario para expresar el efecto mágico que producen estos sonidos jamás escuchados en nuestro planeta. Oyéndolo es dado creer en el más allá", sentenciaba $L a$ Liberté, de la misma capital francesa; pero sin duda la observación más potente fue la de un crítico del New York Herald: "Si Martenot hubiera vivido en la Edad Media, lo habrían procesado por brujería y quemado vivo en la plaza pública". Citas todas ellas bien conocidas por los medios españoles, en las que no solo se inspiraban, sino que en algunos casos reproducían parcialmente para subrayar y enfatizar el carácter especial del acontecimiento. ${ }^{70}$

Obviamente nada había de magia o brujería en las ondas del señor Martenot. Nada más real que aquel aparato que, en todo caso, era producto de la curiosidad y la investigación en el todavía no demasiado trillado campo de la electrónica. Una disciplina que sorprendía con sus principios, aplicaciones y efectos a una sociedad que apenas acertaba a diferenciar su esencia de la pura electricidad. En su narración del primer concierto de las ondas en Madrid, un colaborador del diario $L a$ Voz reconocía: "La electrotecnia es para mí una ciencia de secretos maravillosos e ignorados, que guarda en su seno misterios tan profundos como la alquimia o la necromancia medieval". ${ }^{71}$ En la misma línea, un columnista del diario valenciano Las Provincias escribía: "Como tantas experiencias de orden físico, se saben mejor los efectos que las causas; la electricidad guarda todavía su misterio $[\ldots] "{ }^{72}$

Pero no todos se sentían ajenos a las entrañas de la electrónica ni se dejaron embargar por la apariencia mágica del instrumento. El colaborador del diario El Pueblo, V. Llopis Piquer, daba desde Valencia un enfoque de tinte más

69 La Vanguardia (10.04.1932): 13.

70 La Vanguardia (05.04.1932): 16; (06.04.1932): 10.

71 La Voz (06.04.1932): 3.

72 Las Provincias (13.11.1932): 1. 
real y positivista afirmando con rotundidad que el aparato de monsieur Martenot nada tenía de misterioso ni desconocido. Daba las explicaciones oportunas, hermanaba el instrumento musical con la radio y sus válvulas -para nadie ya producto de magias o brujerías- e incluso facilitaba en una imagen el circuito eléctrico básico de las ondas invitando al lector a su propia construcción y todo -decía cargado de ironía- para que tras el concierto "no quede ninguna duda flotando junto con estas «ondas aéreas» por el espacio". ${ }^{73}$

\section{Bibliografía}

Aracil, Alfredo, Música sobre máquinas y máquinas musicales. Desde Arquímedes a los medios electroacústicos. Madrid, Fundación Juan March, 1984.

Arnaus, Ángels, "Maurice Martenot”, en: Díaz, M. y Giráldez, A. (coords.): Aportaciones teóricas y metodológicas a la educación musical: Una selección de autores relevantes. Barcelona, Grao, 2007, 55-62.

Bloch, Thomas, The Ondes Martenot. Naxos, 2004. [Booklet del CD de Thomas Bloch "Music for ondes Martenot" (ref.: Naxos 8.555779)].

Brice, R., Music engineering: the electronics of playing and recording. Newnes, Butterworth-Heinemann, 2012.

Cage, John, Silence. Madrid, Árdora, 2007.

Cox, Christoph y Warner, Daniel, Audio culture. Readings in modern music. New York, Continuum, 2008.

Gil Noé, José Vicente, “Haciendo historia. Una ¿genealogía? de la experimentación musical y sonora valenciana. 1922-1983", en: Barber, L1.; Palacios, M.; Molina, M., et al.: 100 años de arte sonoro valenciano.
Valencia, Universitat Politècnica de València, 2013, 94-116.

Glinski, Albert, Theremin: ether music and espionage. Chicago, University of Illinois Press, 2000.

González de Posada, Francisco, "Reflexiones en torno al éter", Anales de la Real Academia Nacional de Medicina. Madrid, Real Academia Nacional de Medicina, 2001, Tomo CXVIII-cuaderno 1, 43-72.

Holmes, Thom, Electronic and Experimental Music: pioneers in Technology and Composition. New York, Routledge, 2002.

Holmes, Thom, Electronic and Experimental Music: Technology, Music and Culture. New York, Routledge, 2008.

Lanza, Andrea, Historia de la música. Vol. 12, "El siglo XX”. Madrid, Turner, 1986.

Marco, Tomás, Historia de la música española. Vol. VI, "El siglo XX”. Madrid, Alianza Música, 1983.

Marco, Tomás, Pensamiento musical y siglo XX. Madrid, Fundación Autor, 2002.

Prieberg, Fred K., Música y máquina. Barcelona, Zeus, 1964.

Randel, Don (ed), Diccionario Harvard de Música. Madrid, Alianza, 2001.

Sapena, Sergio, La Sociedad Filarmónica de Valencia (1911-1945): origen y consolidación. Valencia, Tesis doctoral inédita, 2007.

Scholes, Percy A., Diccionario Oxford de la Música. Volumen II. Barcelona, Edhasa, 1984.

Taruskin, Richard, Music in the Late Twentieth Century. New York, Oxford University Press, 2010.

Recibido: 17.04.2013

Aceptado: 02.11.2015

73 El Pueblo (15.11.1932): 3. 